\title{
Graft Copolymerization of Natural Rubber with Maleoyl Chitosan for Antibiosis
}

\author{
Yongzhen LI \\ Key Laboratory of Tropical Crop Products Processing, \\ Ministry of Agriculture of China \\ Agricultural Products Processing Institute of Chinese \\ Academy of Tropical Agriculture Sciences \\ Zhanjiang, China \\ e-mail: 1yz_228@163.com
}

\author{
Mingzhe LV \\ Key Laboratory of Tropical Crop Products Processing, \\ Ministry of Agriculture of China \\ Agricultural Products Processing Institute of Chinese \\ Academy of Tropical Agriculture Sciences \\ Zhanjiang, China
}

\author{
Puwang LI \\ Key Laboratory of Tropical Crop Products Processing, \\ Ministry of Agriculture of China \\ Agricultural Products Processing Institute of Chinese \\ Academy of Tropical Agriculture Sciences \\ Zhanjiang, China \\ e-mail: puwangli@163.com
}

Fan ZHANG

Key Laboratory of Tropical Crop Products Processing, Ministry of Agriculture of China

Agricultural Products Processing Institute of Chinese Academy of Tropical Agriculture Sciences

Zhanjiang, China

\begin{abstract}
Elastomers are necessary materials in building materials. Mouldy materials will not only influence the surface view but also mechanical properties. In this paper, maleoyl chitosan (MCS) was selected as antimicrobial for improving natural rubber (NR) materials' antimicrobial properties together with mechanical and ageing properties. The results demonstrated that MCS showed antimicrobial effects and it also reinforced NR materials in mechanical properties. MCS is of good oil resistivity and thermostability. The copolymerization of them can not only improve the oil resistivity and thermostability of the natural rubber, but also can increase its medicinal value. Benzoyl peroxide (BPO), tertbutyl hydroperoxide (TBHP), dipotassium sulfonatooxy sulfate $\left(\mathrm{K}_{2} \mathrm{~S}_{2} \mathrm{O}_{8}\right)$, azobisisobutyro-nitrile (AIBN), vulcanizing system (sulphur) were selected as initiators. A novel NR-MCS copolymer with much better mechanical property and antibacterial property was obtained at last which would be a good health and medical materials in the future.
\end{abstract}

Keywords-natural rubber; graft copolymerization; maleoyl chitosan

\section{INTRODUCTION}

Elastomers are necessary materials in building materials due to its splice and shockproof functions. Mouldy materials will not only influence the surface view but also mechanical properties. Natural rubber (NR) is got from the plant that can produce rubber latex. It is of excellent chemical and physical properties and can be widely used in many fields, such as: aerospace, war industry, tyre, medical elastomer. Medical elastomer has been used in medical care since the earlier $19^{\text {th }}$ century. The medical elastomer must satisfy the same condition and reach the same goal: it can't hurt the health of the patient and the property must meet the requirement of the application. NR is the first elastomer used in medical care. However, its weak oil resistivity and thermostability restrict its application range.
Chitin is stored richly in the shells of ocean animals such as: shrimp, crab, shellfish and so on. The annual yield of the chitin is about ten billion tons which is just less than that of cellulose. However, chitin is insoluble in most of the solvent, which restrict its application enormously. Chitosan, which is made from the deacetylation of chitin, can be dissolved in the dilute acid. There are much more hydroxyl groups and amino groups in chitosan and it is easy for its structure to be decorated. Chitosan and its derivative are innocuous, biocompatible and biodegradable and can be widely used in biomedical, food, agriculture, cosmetics and environmental protection.

Maleoyl chitosan, which is made from the acylation of chitosan with maleic anhydride using sodium carbonate as catalyst, is a water-soluble polyelectrolyte with good heat resistance. We prepare the grafted copolymer of natural rubber and maleoyl chitosan to improve the oil resistivity and thermostability of the natural rubber. On the other hand, the chitosan is of special physiological and chemical properties, such as: innocuity, good biocompatibility and biodegradability. It also has kinds of pharmacological activities. Therefore, the copolymerization of natural rubber and Maleoyl chitosan may produce a novel polymer material with good medical and pharmacological value.

NR can react with many unsaturated compounds. The reaction of it with maleic anhydride is the earliest and most valuable. Bacon and Farmer found that plasticated NR and maleic anhydride can react in the benzene or toluene using benzoperoxide (BPO) as an initiator at $100^{\circ} \mathrm{C}$ for several hours and got white or buff larinoid product in 1939 [1]. Jia Demin grafted the natural rubber latex with maleic anhydride using BPO as an initiator [2,3]. On the other hand, Zhang Jiping et. al studied on the preparation of maleoyl chitosan and used it as raw material of cosmetics ${ }^{[4]}$. There are also several other researchers prepared maleoyl chitosan with different methods [5-7]. Li Yongzhen et al. 
prepared composite of maleoyl chitosan sodium and nano silica and found that its thermos stability improved strongly [8].

\section{EXPERIMENT}

\section{A. Preparation of Maleoyl Chitosan in Water Medium}

$3.0 \mathrm{~g}$ chitosan mixed uniformly with $300 \mathrm{~mL}$ water at room temperature under stirring, then $1.0 \mathrm{~g}$ sodium carbonate was added. Half an hour later, a certain amount of maleic anhydride was put into the mixture. Six hours later, the reaction was terminated by stopping stirring and 2.0 $\mathrm{mol} / \mathrm{L}$ potassium hydroxide solution was added till the $\mathrm{pH}$ value of mixed solution to 10 . High speed centrifugation was used to separate the rest chitosan from the solution. Hydrochloric acid was added to adjust the $\mathrm{pH}$ value of the supernatant clear liquid after centrifugation. When the $\mathrm{pH}$ value reach to 3.0 , large quantity of white floc was precipitated and separated from the solution by centrifugation again. Then acetone was used to wash the powder until there is no precipitation while silver nitrate solution was added to the washings. The powder was dried in vacuo for 24 hours to get MCS.

\section{B. Copolymerization of NR and MCS}

$0.5 \mathrm{~g}$ MCS was solved in $50 \mathrm{~mL} 10 \%$ (wt \%) ammonia. The solution was drop wised into concentrated natural rubber latex (content of natural rubber is about $60 \%$,wt $\%$ ). Initiator was added in the mixture and the copolymerization happened under proper temperature. After suit time, the mixture after reaction was coagulated with the adding of $\mathrm{NaCl}$. Then the coagulation products were washed with $\mathrm{NaOH}$ solution and water separately. Benzoyl peroxide (BPO), tert-butyl hydroperoxide (TBHP), dipotassium sulfonatooxy sulfate $\left(\mathrm{K}_{2} \mathrm{~S}_{2} \mathrm{O}_{8}\right)$, azobisisobutyro- nitrile (AIBN), vulcanizing system (sulphur) (VS) were used as initiators.

TABLE I. INITIATION REACTION CONDITIONS

\begin{tabular}{|c|c|c|c|c|c|}
\hline Initiator & KPS & TBHP & BPO & AIBN & VS \\
\hline $\begin{array}{c}\text { Reaction } \\
\text { temperature } /\left[{ }^{\circ} \mathrm{C}\right]\end{array}$ & 60 & 60 & 80 & 80 & 60 \\
\hline
\end{tabular}

\section{RESULTS AND DISCUSSIONS}

\section{A. Preparation of MCS}

MCS was prepared from the acylation of maleic anhydride with the amino group of the chitosan. He Jidong gave a hypothetical reaction route of the reaction, but the C6-OH is less reactive than amino group. We modify the ratio of MA and CS to control the graft ratio of MA onto the $\mathrm{CS}$ and restrain the esterification between MA and CS. The ideal graft copolymer to make amphiprotic polyelectrolyte is the MCS whose graft ratio on amino group is about $50 \%$. Then the ratio of amino group and carboxyl group will be 1:1 in the MCS molecular. The main reaction route can be described as in Fig.1.
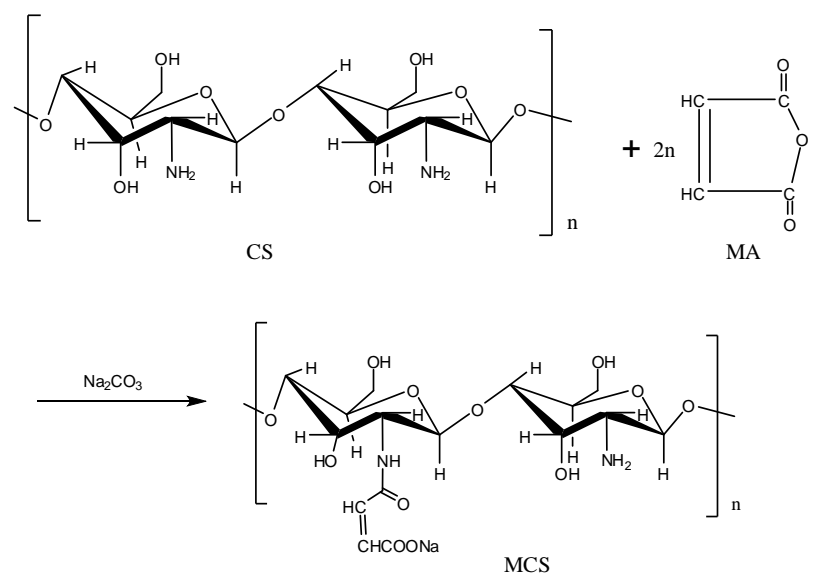

Figure 1. The reaction route of preparing MCS

To get the MCS with graft ratio near 50\%, we investigated the relationship between feed ratio of CS to $\mathrm{MA}$ and graft ratio. The $\mathrm{C}$ percent and $\mathrm{N}$ percent were also tested to calculate the graft ratio.

TABLE II. THE RATIO OF MA to CS AFFECTED TO THE REACTION

\begin{tabular}{|c|c|c|c|c|}
\hline $\mathbf{M}_{\mathbf{M A}}$ : Mcs & Yield/\% & $\begin{array}{c}\text { Graft } \\
\text { ratio/\% }\end{array}$ & $\begin{array}{c}\mathbf{C} \\
\text { percent/\% }\end{array}$ & $\begin{array}{c}\mathbf{N} \\
\text { percent/\% }\end{array}$ \\
\hline $1: 3$ & 10.83 & 23.82 & 44.08 & 7.39 \\
\hline $1: 2$ & 17.05 & 37.51 & 44.56 & 6.93 \\
\hline $1: 1$ & 23.28 & 43.77 & 44.83 & 6.75 \\
\hline $2: 1$ & 41.25 & 50.30 & 45.12 & 6.57 \\
\hline $5: 2$ & 45.27 & 58.62 & 45.61 & 6.38 \\
\hline
\end{tabular}

The yield can be estimated from the weight of MCS $\left(\mathrm{W}_{\mathrm{r}}\right)$ and the weight of MCS we can get from academic complete reaction $\left(\mathrm{W}_{\mathrm{a}}\right)$. Suppose the reaction just happened between carboxyl group of MA and the amino group and there was only one carboxyl group of MA join the reaction.

$$
\text { Yield } / \%=\frac{W_{r}}{W_{a}} \times 100 \%
$$

From the result, we can see the yield is not so high. The possible reason to explain this may come from the purification process of the MCS. MCS can dissolve in both alkaline and acid aqueous solutions while the CS can only dissolve in the acid aqueous solution. We can separate MCS from the residual CS by their different solubility in alkaline aqueous solution. As some of the MCS with low graft ratio can't dissolve in the alkaline aqueous solution completely, they are also removed, so the yield of the reaction may be lower. However, we can make sure that there is no CS in MCS, though the yield is low.

The graft ratio is calculated from the ratio of $\mathrm{C}$ content to $\mathrm{N}$ content $(\mathrm{C} / \mathrm{N})$ which is got from the elementary analysis result. Suppose the MCS is composed of ideal molecules which have no ester function and no cross linkage. The ratio of $\mathrm{C}$ content to $\mathrm{N}$ content $(\mathrm{C} / \mathrm{N})$ can be expressed by the graft ratio(x) in formula (1-2). 


$$
\begin{aligned}
& C / N=\frac{72+48 x}{14} \\
& x=\frac{72-14 \cdot C / N}{48}
\end{aligned}
$$

\section{B. FTIR Characterization of MCS}

The FTIR spectra of CS and MCS give us 3 evidences for the acylation of CS and MA. First, the peaks at $1599 \mathrm{~cm}^{-1}$ and $1657 \mathrm{~cm}^{-1}$ which belong to free amino groups in FTIR spectra of CS move to $1559 \mathrm{~cm}^{-1}$ and $1623 \mathrm{~cm}^{-1}$ respectively which can confirm amide MCS. Second, the wide peak at about $3400 \mathrm{~cm}^{-1}$ which comes from the hydrogen bond caused by free amino groups and hydroxyl in CS become sharper and narrower in MCS. That would be the result of the increase of free amino group. Third, the peak at $3074 \mathrm{~cm}^{-}$ ${ }^{1}$ and $842 \mathrm{~cm}^{-1}$ belong to the $\mathrm{C}=\mathrm{C}$ bond of MA. As the MA has been completely removed from MCS, so the $\mathrm{C}=\mathrm{C}$ bond has been introduced to the MCS molecular successfully. The peak at $1078 \mathrm{~cm}^{-1}$ is the $\mathrm{C}-\mathrm{O}$ of $\mathrm{C}_{6}-\mathrm{OH}$, which can be a proof that most of the $\mathrm{C}_{6}-\mathrm{OH}$ still exist in MCS.

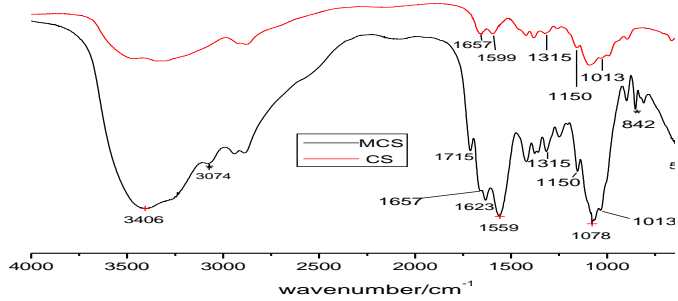

Figure 2. FTIR spectra of CS and MCS

\section{FTIR Characterization of the Copolymers}

Compared the FTIR spectra of the copolymers with the standard spectrum of natural rubber, some new peaks appear in the spectra except most of the absorption peaks in natural rubber. The FTIR absorption peak at $1542 \mathrm{~cm}^{-1}$ is the most obvious. This peak comes from flexural vibration of $\delta \mathrm{N}-\mathrm{H}$ in MCS. This would be a good proof to the copolymerization of NR and MCS.

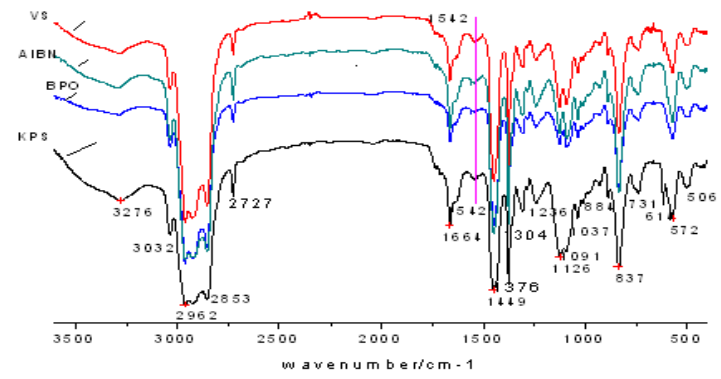

Figure 3. FTIR spectra of the NR-MCS copolymers

\section{Micro Structure Observation}

From the SEM photo of MCS, we can see that the state of MCS is amorphous. As prepared in water suspension, MCS has plenty of holes about $\mu \mathrm{m}$ degree. While in the SEM photo of NR-MCS, the MCS segment is crystal.

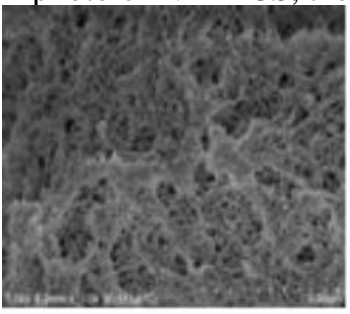

(a) MCS

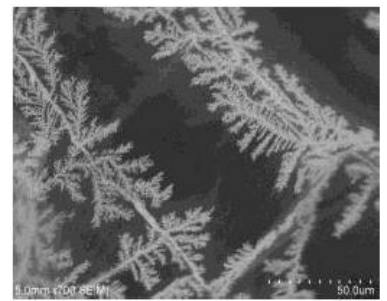

(b) NR-MCS
Figure 4. SEM photos of MCS and NR-MCS

\section{E. Mechanical Property Test}

MECHANICAL PROPERTY TEST RESUlTS OF NR-
MCS COPOLYMERS

\begin{tabular}{|c|c|c|c|c|c|}
\hline & $\begin{array}{c}\text { Tensile } \\
\text { strength } \\
\text { /Mpa }\end{array}$ & $\begin{array}{c}\text { Stress at } \\
100 \% / \mathrm{MPa} a\end{array}$ & $\begin{array}{c}\text { Stress at } \\
300 \% / \mathrm{MPa}\end{array}$ & $\begin{array}{c}\text { Stress at } \\
700 \% / \mathrm{MPa}\end{array}$ & $\begin{array}{c}\text { Elongation } \\
\text { at break/\% }\end{array}$ \\
\hline VS & 41.025 & 0.8516 & 1.451 & 15.310 & 872.312 \\
\hline BPO & 0.3405 & 0.252 & 0.287 & 0.224 & 1028.620 \\
\hline TBHP & 2.245 & 0.582 & 0.835 & 1.531 & 963.025 \\
\hline KPS & 0.431 & 0.344 & 0.385 & 0.412 & 821.667 \\
\hline AIBN & 0.508 & 0.371 & 0.389 & 0.486 & 832.865 \\
\hline
\end{tabular}

The NR-MCS from the initiation of vulcanizing system (sulphur) shows best compositive mechanical property which is much better than that of vulcanized NR. While the copolymers initiated by the other initiators possess even much lower mechanical properties than unmodified NR.

\section{F. Antibacterial Property Test}

From the photos of the washing water antibacterial test samples, there are plenty of bacteria groups grows in the washing water of NR after 48hours of the washing, while no group of bacteria grows in the washing water of NR-MCS. We can say that NR-MCS shows a much better antibacterial property than the NR.

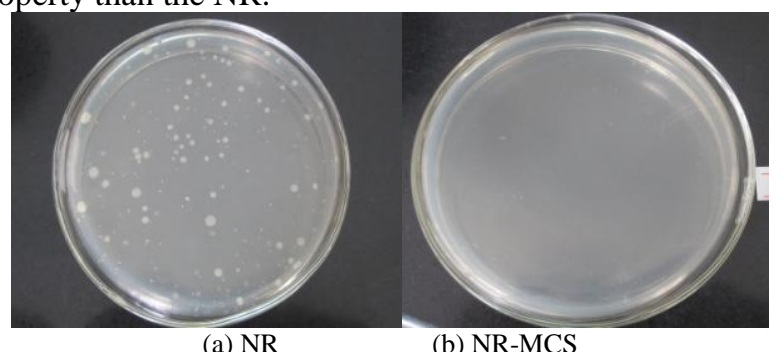

Figure 5. Photos of washing water of NR and NR-MCS after 48-hour test 


\section{CONCLUSIONS}

Acylation of chitosan with maleic anhydride mostly happens between the carboxyl group of MA and the amino group of CS. A novel NR-MCS copolymer is prepared with several initiators. The NR-MCS with vulcanizing system (sulphur) as an initiator shows a much better mechanical property than vulcanized NR from vulcanizing system (sulphur), while other NR-MCS copolymers possess an even worse property than NR. All the copolymers have a good antibacterial property.

\section{ACKNOWLEDGMENT}

This work was financially supported by Major projects in Hainan Province, Critical technique for quality improvement and industry upgrade of natural rubber in Hainan province (ZDKJ2016020) and a grant the basic research fund program (1630022011034) of Rubber Institute, Chinese Academy of Tropical Agriculture Sciences.

\section{REFERENCES}

[1] Bacon R G R, Farmer EH. Rubber Chem. And Technol., 1939, 12(2):200-209.

[2] Dong Zhixian, Jia Demin, Zhou Yanhao et al, China Synthetic Rubber Industry, 2005, 28(3):200-204.

[3] Dong Zhixian, Zhou Yanhao, Tan Lixia et al, Elastomer, 2004, 14(5):1-5.

[4] Zhang Jiping, Qi Yucheng, Liu Yulan et al. China, A61K7/06, CN98110568.

[5] Wang Zhouyu, Jiang Zhenju, Hu Xingqi et al, Applied Chemistry, 2002, 19(10):1002-1004.

[6] Sun Xinzhi, Yang Sheng, Zhang Kaifeng. Chemical Research and Application, 2005, 17(2):243-244.

[7] Ying Guoqing, Yang Hao, Li Donghua et al. Chemical Industry and Engineering Progress, 2007, 26(3):405-408.

[8] Li Yongzhen, He Jidong, Ji Yanfei et al. Science and engineering of polymer materials, 2009, 25(2):164-166. 